Voix et Images

voixetimages

\title{
Vitalités romanesques ou les traditions réinventées
}

\section{Frances Fortier}

Volume 28, numéro 2 (83), hiver 2003

Monique LaRue

URI : https://id.erudit.org/iderudit/006605ar

DOI : https://doi.org/10.7202/006605ar

Aller au sommaire du numéro

\section{Éditeur(s)}

Université du Québec à Montréal

\section{ISSN}

0318-9201 (imprimé)

1705-933X (numérique)

Découvrir la revue

Citer ce compte rendu

Fortier, F. (2003). Compte rendu de [Vitalités romanesques ou les traditions réinventées]. Voix et Images, 28(2), 174-179. https://doi.org/10.7202/006605ar d'utilisation que vous pouvez consulter en ligne.

https://apropos.erudit.org/fr/usagers/politique-dutilisation/ 


\section{Vitalités romanesques ou les traditions réinventées}

\section{Frances Fortier, Université du Québec à Rimouski}

Souvent menacé de suffocation sous les assauts du narcissisme ambiant, parfois croulant sous des dispositifs formels qui confinent à la préciosité, parfois, au contraire, englué dans un quotidien affadi, le roman contemporain ne rend pas les armes pour autant. La fiction narrative, qui vient de renouer avec ce qu'elle avait un temps répudié, à savoir la narration d'une histoire, se réinvente de multiples manières. Trois romans récents, publiés chez Boréal en 2002, en témoignent chacun à sa manière, permettant ainsi une excursion au cœur du divers: tous trois racontent des histoires, plusieurs histoires même, des histoires ciselées au plus près et qui affichent sans complexe ni fausse pruderie leur rapport à la littérature. Le premier relève d'une littérature conçue comme un hommage à l'imaginaire; le second est littéralement un roman de la parole, sur fond de contraintes d'écriture; le troisième, enfin, se réclame du réel, et réinvente le roman de mœurs en lui donnant l'allure d'une fresque murale plaquée sur le visage de la ville.

Music-Hall! de Gaétan Soucy ${ }^{1}$, à l'image de ses romans précédents, propose une anecdote étrange qui se déroule cette fois à New York à la fin des années 1920. Cette fable allégorique, à l'écriture volontiers abstraite malgré la finesse des descriptions, sollicite le lecteur qui, désarçonné par les univers représentés, soup- çonne une énigme. Qui est ce jeune Xavier X. Mortanse, soi-disant émigré hongrois, qui se retrouve un beau jour à travailler comme apprenti sur des chantiers de démolition newyorkais, qui écrit à sa sœur Justine, est fasciné par les joueurs d'échecs et se balade avec une grenouille chantante nommée Strapichacoudou enfermée dans une boîte? Au terme d'épisodes peu ou prou rocambolesques, qui le montrent tabassé par ses collègues démolisseurs ou victime $d u$ public d'un music-hall de bas étage, le mystère se dénoue: Vincent Vilbroquais, le fils de Justine, était venu à New York pour devenir champion aux échecs; devant son insuccès, il s'était défenestré d'un cinquième étage. Justine va l'identifier à la morgue et y retrouve son ancien mari, Rogatien Long-d'Ailes, médecin légiste avec qui elle a vécu dix ans à New York avant de le laisser pour se refaire une vie et avoir cet enfant, Vincent, d'un autre homme mort pendant sa grossesse. Rogatien lui explique tout: Xavier est l'être issu d'une expérience macabre, sorte de Frankenstein bricolé à même des cadavres; son prénom correspond aux initiales des êtres qui le constituent: Xénon, Albert, Vincent, Isabella, Ernest, Reinfeld. L'apprenant, Justine se suicide, non sans avoir transmis à ce Xavier-Vincent le Journal pour une résurrection de Vincent tenu par Long-d'Ailes et qui révèle le détail de ses origines; Xavier meurt 
finalement sur le chantier de démolition, alors que Marie Piquefort, l'enfant chérie du cinéma américain, apparaît sous un cortège d'applaudissements.

L'intérêt du roman, on s'en doute, ne réside pas dans cette ultime révélation, en quelque sorte décevante au plan diégétique. Toutes ces histoires parallèles - celle de Peggy Sue Ohara, celle de Leopold O'Donahue, le Philosophe des Sables du silence, celle de William Cagliari, sorte de Cagliostro maître d'œuvre de l'histoire, celle de Marie Piquefort, «décédée aux mains de Fu-Man-Chou lors de son dernier film » mais qui ressuscite, celle du réalisateur $\mathrm{D}$. W. Griffith - prennent sens à la lumière de cette révélation, apparaissant comme autant de «morceaux » hétéroclites venus d'ailleurs mais qui, à l'image de Xavier X. Mortanse, créent littéralement Music-Hall ! La construction romanesque mime l'histoire, multipliant les mises en abyme et les réduplications, en une sorte de calque du monstrueux Xavier, comme le laissait déjà entrevoir ce numéro de music-hall du «mandarin rafistolé » qui l'a tant effrayé, où un Enchanteur ressuscite un jeune Mandarin «en lui greffant un corps de tigre, deux pattes de chien en guise de jambes, des ailes de pélican, une queue de rat». Les reflets se multiplient au gré des tribulations de la vie de Xavier, qui correspondent par exemple aux titres de certains films de la réelle Mary Pickford réalisés par le non moins réel D. W. Griffith, Willful Peggy, Fauchon The Cricket et Coquette, la «coquette» dont il mourra d'ailleurs. De telle sorte que le roman apparaît, à la relecture, écrit dans la «langue stratakorek", la langue secrète des compagnons démolisseurs. Le foisonnement de péripéties, des numéros abracadabrants de l'autruche Écharlote aux apparitions de la petite Ariane, qui créait le pittoresque du roman, accuse à rebours une toute autre visée: ce bricolage formel de séquences hybrides non seulement mime l'histoire, mais on pourrait affirmer qu'il la mine. L'Ordre américain de la Démolition, employeur de Xavier et qui déblaie l'espace pour construire le «tube», prend ici tout son sens allégorique: le récit démolit littéralement l'histoire, comme l'autruche avale les cadrans-réveil:

Écharlote s'amena de sa démarche caoutchouteuse. Sa patte faisait un bruit de succion en se détachant du sol. Elle portait faux cils, boucles d'oreilles, sa perruque était aussi longue que son cou, et le rouge à lèvres vert pomme qui lui barbouillait le bec était d'un effet insigne. Un nain en costume de clown lui emboîtait le pas. Il poussait une brouette dans laquelle s'amoncelaient une quinzaine de cadrans-réveil, dont les couleurs étaient si vives et diverses qu'on aurait dit un plat de jujubes. [...] Écharlote pencha le bec vers la brouette et, d'un seul coup de gorge, avala un premier cadran. Ce fut dans la salle un rugissement de gaîté méchante. Elle en ingéra un deuxième, puis un autre, ainsi de suite. On entendait l'entrechoquement des cadrans à mesure qu'ils s'engloutissaient dans son ventre. Écharlote s'avança vers la rampe et fixa l'assistance de ses énormes yeux. (p. 273)

Truffé de tels spectacles hallucinants, le monde qui se déploie sous le regard ahuri de Xavier ressemble à s'y méprendre à celui du lecteur contemporain, constamment bombardé d'images hétéroclites qu'il ne sait trop comment décoder. 
Si l'art populaire du music-hall informe l'écriture de Soucy, il faut plutôt chercher du côté de la littérature oulipienne le moteur du dernier roman de Jean-François Chassay, L'angle mort ${ }^{2}$. Virtuose de la contrainte, Chassay multiplie ici les clins d'œil, à commencer par les titres des six parties du roman, empruntés aux Leçons américaines d'Italo Calvino, précision donnée en notice au bénéfice du lecteur. Le salut au maître ne doit pas pour autant masquer les singularités de ce récit à la spécularité emballée, sorte d'EEdipe contemporain qui n'a de cesse de multiplier ses reflets en désignant constamment sa propre structure. Peut-on imaginer un roman vertical ? Trois personnages, dans des espaces distincts l'une dans son bureau au sommet d'un building, l'autre dans la cuisine de son restaurant, le troisième dans un stationnement souterrain qui mène au métro -, monologuent: Stéphane l'architecte parle au téléphone à une amie dont on n'entend pas les réponses, Camille le cuisinier à une interlocutrice anonyme qui l'écoute en silence, l'historien Dominique s'adresse à lui-même (on aura noté les prénoms androgynes).

La simultanéité ajoute encore à l'effet d'étagement; tout le récit se déroule entre $5 \mathrm{~h} 30$ et $7 \mathrm{~h} 45$, en une sorte de prolepse qui prépare les révélations fracassantes à venir. Car chacune de ces histoires en parallèle est construite sur un secret que l'on se propose de divulguer, sans savoir que le mécanisme est réversible. C'est ainsi, par exemple, que Stéphane, la sœur de Dominique, se propose de lui révéler que son fils est de fait l'enfant de Camille tout en ignorant que de son côté Dominique vient lui apprendre que ledit Camille est son propre fils à lui. Le récit s'arrête au seuil de ces divulgations, alors que le frère émerge de l'ascenseur qui l'a mené du sous-sol jusqu'au bureau de sa sœur.

La prouesse réside dans le fait que cet imbroglio, plausible à défaut d'être commun, réussit à donner forme à une réflexion sur l'identité, amorcée dès l'ouverture du roman: «Voyons, dira Stéphane, on construit notre identité grâce aux autres, tu aurais dû apprendre ça en socio. Alors on regarde, on cherche, plus ou moins consciemment, puis on choisit le miroir qui nous convient»(p. 15). Et le texte ne ménagera pas lesdits miroirs, multipliant les réduplications de tous ordres, identitaires, diégétiques, textuelles. Les répliques pullulent, qu'il s'agisse de Camille Moisy et de son double virtuel, homonyme qu'il fréquente par courrier électronique, ou de l'amie Claire qui révélera que sa fille est l'enfant de Stéphane, un Stéphane cette fois masculin il va sans dire. Il n'est jusqu'au roman luimême qui peut apparaître comme un reflet des œuvres antérieures de Chassay, par la reprise, en inversion, de certains personnages et de quelques scènes d'Obsèques ou des Ponts. Constamment, et de mille manières, le texte désigne sa propre forme, sa circularité essentielle, jusque dans les détails les plus anodins, marquant par là une conception du littéraire qui se doit de matérialiser son propos. Écriture ludique certes, néanmoins nourrie d'une lecture de mœurs, qui passe par exemple par les envolées acerbes de la protagoniste Stéphane:

Moi, je préfère appeler un chat un chat, un con un con, un homme un homme, un Juif un Juif, un 
Noir un Noir, une salope une salope, un travail pourri un travail pourri, et la liberté de parole la liberté de parole. Avec tous et toutes, en tout temps et quelle que soit mon humeur. On peut pour ça me traiter de raciste, je répliquerai en traitant, trois fois plus fort, mon interlocuteur de démagogue. Parce que après tout s'il existe quelque chose de politiquement incorrect, c'est bien la démagogie, et s'il existe un type déplorable et d'une insignifiance crasse, c'est bien le démagogue, qui ne se tient pas toujours tranquille à l'intérieur de chacun de nous et qui a depuis longtemps appris à sortir de la bouche des canons des imbéciles. Mon fil, Claire, tu me fais perdre mon fil autobiographique, où en étais-je. Ah oui. (p. 233)

Les angles morts, on l'aura compris dès l'épigraphe, tiré du «Manuel de cours de conduite» «ce sont les espaces périphériques que ne couvrent ni le champ visuel ni les rétroviseurs ». La catastrophe est imminente, elle jaillira de toutes ces filiations imprévisibles et de toutes ces paternités ignorées, fruits d'amours passagères: tel est le tableau que laisse entrevoir L'angle mort, en tournant le miroir vers le passé.

La gloire de Cassiodore de Monique LaRue $^{3}$ tend plutôt le miroir au présent, et montre, en huit chapitres, les aléas d'une année, de la mi-août à mai, dans un collège de Montréal. De la crise personnelle à la crise collective, de l'enthousiasme aveugle aux ambitions trahies, on a là tous les aspects d'un microcosme en ébullition, traversé de luttes syndicales, de désenchantement moral, d'épuisement physique et nerveux, de harcèlement sexuel, un univers de tensions inouïes où les ragots de couloir peuvent tuer et les alliances se reconfigurer au gré d'un cocktail. Le portrait est saisi dans un état de transition, alors que Garneau est au seuil de sa dernière année d'enseignement, à l'heure de dresser une sorte de bilan de sa carrière, de sa vie. Son fils est parti, sa femme vient de le quitter, son meilleur ami est mort à la fin de la session précédente, le jour même de sa retraite. Garneau va mettre par écrit ses réactions, sa conception de la littérature et de l'enseignement, les publier sous le titre «Le journal de Cassiodore » et, du jour au lendemain, devenir une célébrité en accordant une entrevue télévisée. Beau paradoxe, qui ramène Cassiodore sous les feux de l'actualité médiatique :

Flavius Magnus Aurelius Cassiodorus! Consul, préfet sous Théodore, lettré du $\mathrm{VI}^{\mathrm{e}}$ siècle, grand commis de l'État. A écrit une histoire des Goths. Un conciliateur, un penseur du métissage culturel comme on dirait aujourd'hui. Un patricien, amateur de rhétorique. À la fin de sa vie, il se retire dans les terres de sa famille en Calabre. Il fonde un monastère, il organise la copie des textes de l'Antiquité. C'est un pont.

$-\mathrm{Ah}$ !

- Il a écrit un traité de pédagogie. Il a défini les savoirs élémentaires. Savoir lire, savoir compter.

- Ah ah. (p. 128-129)

Monique LaRue est une écrivaine engagée, si tant est que le terme puisse encore être entendu. Son récit, rondement mené, à l'écriture vive et maîtrisée, de facture réellement romanesque aurait-on envie de dire, donne forme à toute une suite de polarisations qui traversent les milieux de l'enseignement littéraire. Structuré par les indications du pro- 
gramme qui le partagent en deux parties - «De Rabelais à Laclos avant Noël» et «De Hugo à Camus après Noël»-, le roman, par l'entremise de personnages emblématiques, met en scène les clivages qui départagent, entre autres, les anciens des modernes, les premiers héritiers de la tradition classique et les seconds, comme la jeune professeure Pétula Cabana, qui défendent bec et ongles la création littéraire sur fond d'autofiction. Le clan des anciens n'est pas pour autant homogène, même si tout ce beau monde, à une époque pas si lointaine, vivait dans l'exubérance des amours adultères, quasi incestueuses, où le mari de l'une devenait l'amant de l'autre: ils ont maintenant devant eux les enfants de leurs collègues, parfois même les leurs, qu'ils arrivent difficilement à rejoindre. Et le récit de multiplier ainsi les lignes de fracture, au gré des aspirations des personnages: l'un est devenu administrateur scolaire, l'autre délégué syndical; un autre s'est crispé dans son intransigeance, faisant échouer la majorité de ses étudiants alors qu'un autre encore les recueille chez lui.

La nouvelle génération de professeurs mènera d'autres combats, écartelée qu'elle sera entre l'enseignement universitaire et l'enseignement collégial, entre le milieu scolaire et le milieu de l'édition; même les étudiants sont divisés, les produits de l'école alternative, «l'École naturelle des cormorans», méprisant royalement ceux issus de la formation publique. La vie quotidienne du collège, cependant, va peu à peu sinon dissoudre ces antagonismes, du moins les reconfigurer autrement; la vitalité du récit réside précisément dans cet art de la nuance qui vient gommer les aspérités par trop évidentes et ménager des terrains d'entente, à l'image du bureau partagé par Garneau et Cabana. Une telle sotie ne saurait laisser indifférent quiconque s'interroge sur la nécessité de la littérature, sur le désenchantement, les illusions perdues.

Ces trois romans ont su trouver leur public, comme en témoigne la réception qui a suivi leur parution. Serait-ce qu'ils savent incarner les interrogations prégnantes de notre époque? Leur posture critique, car c'est bien de cela qu'il s'agit, se fait à la fois éthique, herméneutique et esthétique. La gloire de Cassiodore met en scène la lancinante question de l'enseignement littéraire en montrant concrètement les coulisses du collège, et agit comme un révélateur des modalités complexes de la transaction générationnelle et de la difficile transmission des valeurs. L'acuité du regard n'épargne personne, ni les «boy-scouts de l'enseignement»ni les «sophistes» qui prétendent rentabiliser l'école; il ne s'agit pas pour autant de juger, mais bien d'essayer de comprendre la faillite d'une société qui ne sait pas toujours contrer la médiocrité ambiante. À l'image de la fresque peinte par le fils de Garneau et présentée dès l'ouverture, le roman met à plat, dans un désordre savamment orchestré, les engagements individuels. Le propos est sans nul doute plus acerbe dans L'angle mort. Les personnages vitupèrent constamment contre l'omniprésence des téléphones cellulaires, la vanité des combats collectifs, la schizophrénie de nos comportements, la lourdeur des héritages familiaux. L'individualisme ne saurait mieux s'incarner 
que dans ce dispositif formel qui monte trois histoires en parallèle, à la faveur de monologue: les trois personnages s'adressent à quelqu'un qui ne répond pas, chacun enfermé dans un soliloque interminable qui ne trouvera pas sa résolution dans le cadre du roman. Pourtant, on le pressent, ces individualistes seront torpillés précisément par la teneur des secrets, qui vont les anéantir précisément par ce qu'ils dénoncent, en tressant entre eux des liens inextricables. Enfin, Music-Hall! peut se lire comme une réflexion sur l'identité postmoderne, qui ne saurait différencier la réalité de la fiction. Xavier X. Mortanse, à la fois acteur et spectateur de sa vie, la voit se dérouler comme au spectacle, en une suite de vignettes tout aussi farfelues et incompréhensibles les unes que les autres. Que représente, sinon chacun d'entre nous, ce pantin désarticulé de music-hall qui se bricole une existence factice, se l'invente littérale- ment, à partir d'une affiche de voyage et d'une vieille photographie posées par hasard à sa portée?

Ces romans hybrides, imprégnés de culture populaire, d'arts mineurs comme de réflexions savantes, sont portés d'un pareil souffle: il faut raconter, raconter pour libérer l'imagination, raconter pour comprendre, raconter pour créer un monde plus vivable, raconter pour donner sens à l'insensé, raconter pour interroger le présent, raconter pour survivre. «Tous ceux qui entouraient Garneau étaient sans exception convaincus que la capacité de déplier les délicats feuillets d'un roman est en tous points indispensable à l'humain pour rester humain" (p. 295). On ne saurait mieux dire.

1. Gaétan Soucy, Music-Hall !, Montréal, Boréal, 2002, 390 p.

2. Jean-François Chassay, L'angle mort, Montréal, Boréal, 2002, 333 p.

3. Monique LaRue, La gloire de Cassiodore, Montréal, Boréal, 2002, 297 p.

\section{Poésie}

\section{Ici, c'est ailleurs}

\section{André Brochu, Université de Montréal}

Des écrits de prime jeunesse de Saint-Denys Garneau, les poèmes et de fulgurants souvenirs en prose de Michel van Schendel, les actes d'un colloque consacré à Gaston Miron composent le programme de cette chronique. L'on y examinera quelques façons d'être ailleurs en étant d'ici, ou l'inverse.

\section{Le pré-poète}

Giselle Huot, bonne cuisinière, n'en finit plus d'apprêter l'œuvre de Saint-Denys Garneau à toutes les sauces, multipliant éditions critiques, anthologies et - c'est le cas aujourd'hui - publication d'inédits. Or le Recueil de poésies - Inédit de $1928^{1}$ ne révèle rien de neuf puisque les 